\title{
ON THE REALIZATION AND CLASSIFICATION OF CYCLIC EXTENSIONS OF POLYNOMIAL ALGEBRAS OVER THE STEENROD ALGEBRA
}

\author{
HOWARD HILLER AND LARRY SMITH
}

\begin{abstract}
Suppose $\mathbf{R}^{*}$ is an unstable algebra over the Steenrod algebra of the form $\mathbf{P}^{*}(\sqrt[k]{d})$, where $\mathbf{P}^{*}$ is a polynomial algebra over the Steenrod algebra. If $\mathbf{R}^{*}$ is integrally closed then $\mathbf{R}^{*}=P(V)^{G_{x}}$, where $C \leqslant G L(V)$ is generated by pseudoreflections and $G_{\chi}=\operatorname{ker}\left\{\chi: G \rightarrow \mathbf{F}_{p}^{*}\right\}$ is a character of degree $k$.
\end{abstract}

Let $\mathbf{P}^{*}$ be an unstable polynomial algebra over the Steenrod algebra. By a cyclic, or radical, extension of $\mathbf{P}^{*}$ of degree $k$ we mean an unstable algebra $\mathbf{R}^{*}$ over the Steenrod algebra containing $\mathbf{P}^{*}$ as a subalgebra and closed under the action of the Steenrod algebra such that: $\mathbf{R}^{*}$ is generated as an algebra by $\mathbf{P}^{*}$ and a class $f \in \mathbf{R}^{*}$ such that the minimal polynomial of $f$ over $\mathbf{P}^{*}$ is of the form $Y^{k}-d$ for some $d \in \mathbf{P}^{*}$. In other words $\mathbf{R}^{*} \cong \mathbf{P}^{*}(\sqrt[k]{d})$ as algebras over the Steenrod algebra.

Let $V=\oplus_{n} \mathbf{F}_{p}, G \leqslant G L(V)$ and $\chi: G \rightarrow \mathbf{F}_{p}^{*}$ a linear character of $G$ of order $k$. Following Stanley [6] define

$$
P(V)_{\chi}^{G}=\{h \in P(V) \mid g h=\chi(g) h \forall g \in G\} .
$$

This is the $P(V)^{G}$-module of $\chi$-relative invariants. Set

$$
G_{\chi}:=\operatorname{ker}\left\{\chi: G \rightarrow \mathbf{F}_{p}^{*}\right\}
$$

and note $G / G_{x} \cong \mathbf{Z} / k$. If $G$ is generated by pseudoreflections and of order relatively prime to $p$ then the nonmodular characteristic $p$ version of [6] says that there exists a class $f_{\chi} \in P(V)$, which is a product of linear forms, such that

$$
P(V)_{\chi}^{G} \cong P(V)^{G} f_{\chi}: f_{\chi}^{k} \in P(V)^{G}
$$

and

$$
P(V)^{G} \cong \bigoplus_{i=0}^{k-1} P(V)^{G} f_{\chi} i .
$$

There is the coexact extension sequence

$$
\mathbf{F}_{p}^{*} \rightarrow P(V)^{G} \rightarrow P(V)^{G} \chi \rightarrow P(V)_{G}^{\chi} \rightarrow F_{p}^{*}
$$

Received by the editors May 1, 1985 and, in revised form, February 15, 1986.

1980 Mathematics Subject Classification (1985 Revision). Primary 55S10, 13 B10. 
defining $P(V)_{G}^{\chi} \cong P(V)^{G} / / P(V)^{G}$. The algebra $P(V)_{G}^{\chi}$ has the following structure:

$$
\begin{gathered}
P(V)_{G}^{\chi} \cong \bigoplus_{i=0}^{k} f_{\chi} i \cdot F_{p}, \\
f_{\chi} r \cdot f_{\chi} s= \begin{cases}f_{\chi} t & \text { if } \operatorname{deg} f_{\chi} r+\operatorname{deg} f_{\chi} s=\operatorname{deg} f_{\chi} t \\
0 & \text { otherwise. }\end{cases}
\end{gathered}
$$

A special case of this situation is when

$$
f_{\chi} i=\left(f_{\chi}\right)^{i} \in P(V): i=0, \ldots, k-1 .
$$

In this case we say that $P(V)^{G} \geqslant P(V)^{G}$ is a cyclic extension of $P(V)^{G}$ of degree $k$. If $d=f_{x}^{k}$ then we write $P(V)=P(V)^{G}(\sqrt[k]{d})$ in this case.

Here is a simple example.

EXAMPLE. Let $p$ be an odd prime, $s$ a divisor of $p-1$, and $D(2 s)=\mathbf{Z} / s \ltimes \mathbf{Z} / 2$ the dihedral group of order $2 s$. Embed $D(2 s)$ in $G L\left(2, \mathbf{F}_{p}\right)$ by choosing the matrices

$$
\left[\begin{array}{cc}
\Theta^{r} & 0 \\
0 & \Theta^{r}
\end{array}\right],\left[\begin{array}{ll}
0 & 1 \\
1 & 0
\end{array}\right]
$$

as generators, where $\Theta \in F_{p}^{*}$ is a generator and $r=(p-1) / s$. Then

$$
P[x, y]^{D(2 s)}=P\left[x y, x^{s}+y^{s}\right] \text {. }
$$

Let $\Delta: D(2 s) \rightarrow F_{p}{ }^{*}$ be the determinant character. Then $D(2 s)_{\Delta}=\operatorname{ker} \Delta=\mathbf{Z} / s$. Note

$$
P[x, y]_{\Delta}^{D(2 s)}=P[x, y]^{D(2 s)} \cdot f_{\Delta}
$$

where $f_{\Delta}=x^{s}-y^{s}$, and

$$
P[x, y]^{D(2 s)_{\Delta}}=P[x, y]^{\mathrm{Z} / s}=P\left[x^{s}, y^{s}, x y\right] /\left(x^{s} y^{s}-(x y)^{s}\right) .
$$

In this case $P(V)^{D(2 s)_{\Delta}}$ is in fact a quadratic extension of $P(V)^{D(2 s)}$, namely

$$
P(V)^{D(2 s)_{\Delta}}=P(V)^{D(2 s)}(\sqrt{d})
$$

where $d=\left(x^{s}-y^{s}\right)^{2}$.

One of our goals is to prove a converse to Stanley's theorem. We begin, however, with a discussion of an alternate construction procedure for radical extensions. This in turn is based on the observation that, if we place ourselves in the nonmodular characteristic $p$ situation of Stanley's theorem, where

$$
P(V)_{\chi}^{G}=P(V)^{G} f_{\chi},
$$

and recall that $P(V)^{G_{x}}$ is closed under the action of the Steenrod algebra, then

$$
\mathscr{P}\left(f_{\chi}\right)=w f_{\chi}
$$

for some ungraded class $w=1+w_{1}+w_{2}+\cdots \in P(V)^{G}$, where

$$
\mathscr{P}=1+P^{1}+P^{2}+\cdots
$$

is the total Steenrod operation. In other words, $f_{\chi}$ is an eigenvector of the automorphism $\mathscr{P}$. Of course, this follows from Stanley's result that $f_{\chi}$ is a product of linear forms. Moreover, the eigenvalue $w$ lies in $P(V)^{G}$. It is less apparent that 
this follows from Stanley's result. Surprisingly enough, the converse of all this holds. To make this precise we introduce a definition.

Definition. $d \in P(V)$ is called special iff there exists $w=1+w_{1}+w_{2}+\cdots \in$ $P(V)$ such that $\mathscr{P}(d)=w d$.

THEOREM 1. $d \in P(V)$ is special iff $d$ is a product of two-dimensional classes.

The proof of Theorem 1 is based on the following result of Landweber [5].

THEOREM (LANDWEBER). Let $\mathbf{H}^{*}$ be an unstable algebra over the Steenrod algebra. Assume $\mathbf{H}^{*}$ is Noetherian. Then the associated prime ideals of $\mathbf{H}^{*}$ are invariant under the Steenrod algebra.

There are several different ways to define the associated primes of $\mathbf{H}^{*}$. For our purposes we take them to be the prime ideals associated to an irredundant primary decomposition of zero in $\mathbf{H}^{*}$.

Here is a sketch of Landweber's proof. Introduce the total Steenrod operation in the form

$$
\mathscr{P}_{T}: \mathbf{H}^{*} \rightarrow \mathbf{H}^{*}[T]: \operatorname{deg} T=-2(p-1)
$$

where

$$
\mathscr{P}_{T}(h)=\sum_{i=0}^{\infty} \mathscr{P}^{i}(h) T^{i} .
$$

Note that $\mathscr{P}_{T}$ is a homogeneous (degree-preserving) algebra injection. Let

$$
0=q_{1} \cap \cdots \cap q_{\Gamma}: p_{i}=\sqrt{q_{i}}, \quad i=1, \ldots, r
$$

be an irredundant primary decomposition of zero in $\mathbf{H}^{*}$. This extends up to the irredundant primary decomposition of zero

$$
0=q_{1}[T] \cap \cdots \cap q_{\Gamma}[T]
$$

in $\mathbf{H}^{*}[T]$. Since $\mathscr{P}_{T}$ is injective this gives a primary decomposition of zero in $\mathbf{H}^{*}$

$$
0=\mathscr{P}_{T}^{-1}\left(q_{1}[T]\right) \cap \cdots \cap \mathscr{P}_{T}^{-1}\left(q_{\Gamma}[T]\right)
$$

with associated primes

$$
\mathscr{P}_{T}^{-1}\left(\not_{i}[T]\right)=\left\{x \in H^{*} \mid P^{k}(x) \in \not h_{i} \forall k \geqslant 0\right\} .
$$

Note

$$
\mathscr{P}_{T}^{-1}\left(\not h_{i}[T]\right) \subset \not \mathfrak{h}_{i} \quad \forall i .
$$

By uniqueness of associated primes for irredundant primary decompositions (Lasker-Noether) it follows that

$$
\mathscr{P}_{T}^{-1}\left(\not_{i}[T]\right)=\not_{i}, \quad i=1, \ldots, r .
$$

But this says $h_{i}$ is invariant, as wished.

As an easy consequence of Landweber's theorem we obtain (N. B. By an invariant ideal we mean an ideal invariant under the action of the Steenrod algebra):

COROLlaRY 2. Let $\mathbf{R}^{*}$ be an unstable Noetherian algebra over the Steenrod algebra, and $\mathscr{I}$ an invariant ideal. If

$$
\mathscr{I}=q_{1} \cap \cdots \cap q_{r}
$$


is an irredundant primary decomposition of $\mathscr{I}$ in $\mathbf{R}^{*}$, then $\mu_{i}=\sqrt{q_{i}}, i=1, \ldots, r$, are invariant prime ideals of $\mathbf{R}^{*}$.

Proof. Pass to $\mathbf{R}^{*} / \mathscr{I}$, apply Landweber's result, and lift back.

Proof of Theorem 1. One direction is clear. Namely, if $d=\prod_{i=1}^{s} x_{i}, \operatorname{deg} x_{1}=$ $\cdots=\operatorname{deg} x_{s}=2$, then

$$
\mathscr{P} d=d \prod_{i=1}^{s}\left(1+x_{i}^{p-1}\right),
$$

so $d$ is special. Conversely, suppose that

$$
\mathscr{P} d=w d: w \in P(V) .
$$

Then the principal ideal $(d) \subset P(V)$ is invariant. Let

$$
d=p_{1}^{e_{1}} \cdots p_{r}^{e_{r}}
$$

be a factorization of $d$ into a product of relatively prime irreducible polynomials. Then

$$
(d)=\left(p_{1}^{e_{1}}\right) \cdots\left(p_{r}^{e_{r}}\right)=\left(p_{1}^{e_{1}}\right) \cap \cdots \cap\left(p_{r}^{e_{r}}\right)
$$

is an irredundant primary representation of $(d)$ by primary ideals. By the Corollary to Landweber's theorem $\left(p_{1}\right), \ldots,\left(p_{r}\right)$ are invariant prime ideals. But by the theorem of Serre and Wilkerson [7] the invariant prime ideals in $P(V)$ are generated by two-dimensional classes. Therefore $\operatorname{deg} p_{i}=2, i=1, \ldots, r$.

Corollary 3. If $d \in P(V)$ is special, and $f \in P(V)$ is such that $f^{k}=d$, then $f$ is special.

Proof. By Theorem $1, d$ is a product of linear forms. $P(V)$ is a unique factorization domain and therefore $f$ is also a product of linear forms and hence special.

Definition. Let $f \in P(V)$ and $\mathbf{P}^{*} \leqslant P(V)$ be a subalgebra closed under the action of the Steenrod algebra. We say $f$ is $\mathbf{P}^{*}$-special $\Leftrightarrow \mathscr{P} f=w f$ with $w \in \mathbf{P}^{*}$.

We are indebted to Clarence Wilkerson for the following two results, which replace less general results of our own.

Proposition 4. Suppose $\mathbf{P}^{*} \leqslant P(V)$ is closed under the action of the Steenrod algebra. Assume $\mathbf{P}^{*}$ is integrally closed and $P(V)$ is finite over $\mathbf{P}^{*}$. If $d \in \mathbf{P}^{*}$ is $P(V)$-special, then it is $\mathbf{P}^{*}$-special.

Proof. We have

$$
\mathscr{P} f=w f: w \in P(V) .
$$

But $w=\mathscr{P} f / f$ belongs to the field of fractions of $\mathbf{P}^{*}$. Since $P(V)$ is finite over $\mathbf{P}^{*}$ the elements of $P(V)$ are integral over $\mathbf{P}^{*}$. Therefore the homogeneous components of $w$ are integral over $\mathbf{P}^{*}$ and since $\mathbf{P}^{*}$ is integrally closed must be contained in it.

RemarK. If $\mathbf{P}^{*}=P(V)^{G}, G \leqslant G L(V)$, then $\mathbf{P}^{*}$ satisfies the hypotheses of Proposition 4. 
Proposition 5. Suppose $\mathbf{P}^{*} \leqslant P(V)$ is closed under the action of the Steenrod algebra and $P(V)$ is finite over $\mathbf{P}^{*}$. Assume $f \in P(V)$ is $P(V)$-special. If $f^{k} \in \mathbf{P}^{*}$, $(k, p)=1$, then $f$ is $\mathbf{P}^{*}$-special.

Proof. We need to show $\mathscr{P}^{i} f / f \in \mathbf{P}^{*} \forall i$. In fact we will show inductively that $\mathscr{P}^{i} f / f$ is a polynomial in $\mathscr{P}^{1} d / d, \ldots, \mathscr{P}^{i} d / d$. By Proposition 4 the latter are in $\mathbf{P}^{*}$. We begin with the equations

$$
\mathscr{P}_{T}\left(f^{k}\right) / f^{k}=\left(\frac{\mathscr{P}_{T}(f)}{f}\right)^{k}=1+\frac{\mathscr{P}^{1} f}{f} T+\cdots+f^{p-1} T^{N},
$$

where $N=-2 \operatorname{deg}(f)(p-1)$ and

$$
\text { (\# \#) }
$$

$$
\frac{\mathscr{P}_{T}\left(f^{k}\right)}{f^{k}}=\frac{\mathscr{P}_{T}(d)}{d}=1+\frac{\mathscr{P}^{q} f}{d} T+\cdots+d^{p-1} T^{k N} .
$$

Equating gives

$$
\left(1+\frac{\mathscr{P}^{1} f}{f}+\cdots+f^{p-1} T^{N}\right)^{k}=1+\frac{\mathscr{P}^{1} d}{d} T+\cdots+d^{p-1} T^{k N}
$$

from which follows

$$
k \frac{\mathscr{P}^{1} f}{f}=\frac{\mathscr{P}^{1} d}{d}
$$

and since $k$ is a unit $\bmod p$,

$$
\frac{\mathscr{P}^{1} f}{f}=k^{-1} \frac{\mathscr{P}^{1} d}{d} .
$$

This allows us to start an induction. From $(*)$ we get

$$
k \frac{\mathscr{P}^{j} f}{f}+G_{j}\left(\frac{\mathscr{P}^{1} f}{f}, \ldots, \frac{\mathscr{P}^{j-1} f}{f}\right)=F_{j}\left(\frac{\mathscr{P}^{1} d}{d}, \ldots, \frac{\mathscr{P}^{j} d}{d}\right) .
$$

By induction $G_{j}()$ and $F_{j}() \in \mathbf{P}^{*}$, and since $k$ is a unit $\bmod p$ the result follows.

As a consequence of this discussion we obtain the following procedure for constructing cyclic extensions.

Suppose $G \leqslant G L(V)$, and $d \in P(V)^{G}$ is special. If $d$ has a $k$ th root $f$ in $P(V)$, where $k$ is a divisor of $p-1$. Then

$$
\mathscr{P} f=u f, \quad u \in P(V)^{G} .
$$

Therefore $\mathbf{R}^{*}$ is the subalgebra of $P(V)$ generated by $P(V)^{G}$ and $f, \mathbf{R}^{*}$ is closed under the action of the Steenrod algebra. Therefore $\mathbf{R}^{*}$ is a radical extension of $P(V)^{G}$. Note that the degree of the extension is $k^{\prime}$ where $k^{\prime}$ is the smallest divisor of $k$ such that $f^{k^{\prime}} \in P(V)^{G}$. Finally, note that to check whether $d$ has a $k$ th root in $P(V)$ is an easy task, because by Theorem $1 d$ is a product of linear forms. If we write

$$
d=v_{1}^{e_{1}} \cdots v_{r}^{e_{r}}
$$


with $v_{1}, \ldots, v_{r} \in V$ relatively prime linear forms, then $d$ has a $k$ th root iff $e_{i} \equiv 0$ $\bmod k, i=1, \ldots, r$.

We next prove the converse of Stanley's theorem in the nonmodular characteristic $p$ setting. We require some definitions.

Definition. Let $\mathbf{T}^{*}$ be a graded connected totally finite algebra over $\mathbf{F}_{p}$. We say $T^{*}$ has maximally nonzero multiplication iff

$$
\operatorname{dim} T^{j} \leqslant 1 \text { and } \mu: T^{i} \otimes T^{j} \rightarrow T^{i+j} \text { is surjective. }
$$

Note that this is equiuvalent to saying that $\mathbf{T}^{*}$ has a basis $1=f_{0}, \ldots, f_{k}, k=$ $\operatorname{dim} \operatorname{Tot}\left(T^{*}\right)$, where

$$
f_{r} \cdot f_{s}= \begin{cases}f_{t} & \text { if } \operatorname{deg}\left(f_{r}\right)+\operatorname{deg}\left(f_{s}\right)=\operatorname{deg}\left(f_{t}\right), \\ 0 & \text { otherwise. }\end{cases}
$$

Definition. Let $\mathbf{P}^{*}$ be an unstable polynomial algebra over the Steenrod algebra. A cyclic extension of $\mathbf{P}^{*}$ of degree $k$ is a coexact sequence

$$
\mathbf{F}_{p} \rightarrow \mathbf{P}^{*} \rightarrow \mathbf{R}^{*} \rightarrow \mathbf{T}^{*} \rightarrow \mathbf{F}_{p},
$$

where $\mathbf{R}^{*}$ is a free $\mathbf{P}^{*}$ of rank $k$, and $\mathbf{T}^{*}$ is a faithful $\mathbf{Z} / k$ algebra with maximally nonzero multiplication. (N.B. If $H$ is a group, then an $H$-algebra is an algebra over the Hopf algebra $\mathbf{F}_{p}[H]$. It is faithful if the action of $H$ is faithful. Note that $P[f] /\left(f^{k}\right)$, where $k$ is a divisor of $p-1$, is a $\mathbf{Z} / k$ algebra in a natural way, where the action of a generator $\gamma$ of $\mathbf{Z} / k$ is given by:

$$
\gamma f=\Theta^{m} f: m=(p-1) / m
$$

and $\theta$ is a primitive $(p-1)$ st root of unity. This is the situation when $\mathbf{R}^{*}=\mathbf{P} *(\sqrt[k]{d})$.)

TheOREM 6. Let $\mathbf{R}^{*} \geqslant \mathbf{P}^{*}$ be a cyclic extension of degree $k, k \mid p-1$, where $\mathbf{R}^{*}$ and $\mathbf{P}^{*}$ are generated by classes of degrees relatively prime to $p, \mathbf{P}^{*}$ is a polynomial algebra and $\mathbf{R}^{*}$ is integrally closed. Then there exists $G \leqslant G L(V)$ generated by pseudoreflections of order relatively prime to $p$ and a linear character $\chi: G \rightarrow \mathbf{F}_{p}^{*}$ of degree $k$ such that

$$
\begin{array}{ccc}
\mathbf{R}^{*} & \simeq P(V)^{G_{x}} \\
\vee & \vee \\
\mathbf{P}^{*} & \simeq P(V)^{G} .
\end{array}
$$

Proof. By hypothesis $\mathbf{R}^{*}$ is integrally closed and therefore we may apply [1] to embed $\mathbf{R}^{*} \hookrightarrow P(V)$, for some $V$, with $P(V)$ the algebraic closure of $\mathbf{R}^{*}$ in the category of unstable domains over the Steenrod algebra. Since $\mathbf{P}^{*}$ and $\mathbf{R}^{*}$ have the same transcendence degree, the composite embedding $\mathbf{P}^{*} \hookrightarrow \mathbf{R}^{*} \hookrightarrow P(V)$ is the embedding of $\mathbf{P}^{*}$ into its algebraic closure also. Therefore by $[\mathbf{1}] \mathbf{P}^{*}=P(V)^{G}$ for some $G \leqslant G L(V)$ generated by pseudoreflections of order relatively prime to $p$. Let

$$
\mathbf{R}^{*}=\bigoplus_{i=0}^{k-1} \mathbf{P}^{*} \cdot f_{i}
$$

and identify $\left\{1=f_{0}, \ldots, f_{k-1}\right\}$ with a basis for $\mathbf{T}^{*}$. This defines an action of $\mathbf{Z} / k$ on $\mathbf{R}^{*}$. Let $\gamma \in \mathbf{Z} / k$ be a generator. Suppose

$$
r=h_{0}+h_{1} f_{1}+\cdots+h_{k-1} f_{k-1}
$$


and $\gamma r=r$. Then

$$
h_{0}+h_{1} f_{1}+\cdots+h_{k-1} f_{k-1}=h_{0}+h_{1} \gamma f_{1}+\cdots+h_{k-1} \gamma f_{k-1} .
$$

But $\left\{1=f_{0}, f_{1}, \ldots, f_{k-1}\right\}$ is a basis for $\mathbf{R}^{*}$ over $\mathbf{P}^{*}$ and hence

$$
f_{i}=\gamma f_{i}: i=1, \ldots, k-1
$$

and since $\mathbf{T}^{*}$ is faithful this means

$$
h_{1}=h_{2}=\cdots=h_{k-1}=0,
$$

so $r \in \mathbf{P}^{*}$. Thus $\mathbf{R}^{*}$ is Galois over $\mathbf{P}^{*}$ with Galois group $\mathbf{Z} / k$. Let $F()$ be the field of fractions functor. Then we have

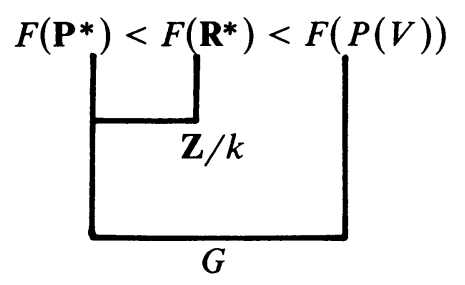

By the fundamental theorem of Galois theory $F\left(\mathbf{R}^{*}\right) \leqslant P(V)$ is Galois with Galois group $H \triangleleft G$ and $G / H \simeq \mathbf{Z} / k$. If

$$
\chi: G \rightarrow G / H \simeq \mathbf{Z} / k<\mathbf{F}_{p}^{*}
$$

is the composite linear character, we have $H=G_{\chi}$. Since everything in sight is integrally closed we may lift to obtain

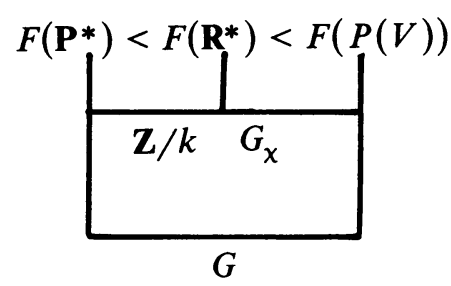

as required.

ACKNOWLEDGments. We wish to thank John Ewing for discussions that led to Theorem 1, and Peter Landweber, R. E. Stong, and Clarence Wilkerson for suggestions that greatly improved the exposition. We wish to thank R. J. Milgram for the word processing program that made possible the problem-free typing of this paper.

\section{REFERENCES}

1. J. F. Adams and C. W. Wilkerson, Finite $H$-spaces and algebras over the Steenrod algebra, Ann. of Math. 78 (1980), 95-143.

2. N. Bourbaki, Groupes et algèbres de Lie. V. Hermann, Paris, 1968.

3. C. Chevalley, Invariants of finite groups generated by reflections, Amer. J. Math. 78 (1955), 778-782.

4. H. Hiller, The geometry of Coxeter groups, Pitman, 1982.

5. S. Landweber, Dickson invariants and prime ideals invariant under Steenrod operations, Rutgers University Preprint, 1984. 
6. R. P. Stanley, Relative invariants of finite groups generated by pseudoreflections, J. Algebra 49 (1977), 134-148.

7. C. W. Wilkerson, Classifying spaces, Steenrod operations and algebraic closure, Topology 16 (1977), $227-237$.

Department of Mathematics, Columbia University, New York, New York 10027

Mathematisches Institut, Georg Augustus Universität, D3400 GötTingen, West Germany (Current address of Larry Smith)

Current address (Howard Hiller): Citicorp Investment Bank, New York, New York 10043 